\title{
Simultaneous X-ray and optical observations of S5 0716+714 after the outburst of March 2004^
}

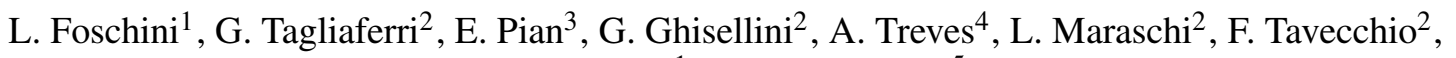 \\ G. Di Cocco ${ }^{1}$, and S. R. Rosen ${ }^{5}$
}

\author{
1 INAF/IASF - Bologna, via Gobetti 101, 40129 Bologna, Italy \\ e-mail: foschini@iasfbo.inaf.it \\ 2 INAF - Osservatorio Astronomico di Brera, via Brera 28, 20121 Milano, Italy \\ 3 INAF - Osservatorio Astronomico di Trieste, via Tiepolo 11, 34131 Trieste, Italy \\ 4 Dipartimento di Scienze, Università degli Studi dell'Insubria, via Valleggio 11, 22100 Como, Italy \\ 5 Mullard Space Science Laboratory, University College of London Holmbury St Mary, Dorking, Surrey, RH5 6NT, UK
}

Received 3 February 2006 / Accepted 26 April 2006

\begin{abstract}
At the end of March 2004, the blazar S5 $0716+714$ underwent an optical outburst that prompted for quasi-simultaneous target-ofopportunity observations with the INTEGRAL and XMM-Newton satellites. In this paper, we report the results of the XMM-Newton and INTEGRAL OMC data analysis. The X-ray spectrum is well-represented by a concave broken power-law model, with the break at about $2 \mathrm{keV}$. In the framework of the synchrotron self-Compton model, the softer part of the spectrum, which is described by a power law of index $\alpha \simeq 1.8\left(f_{v} \propto v^{-\alpha}\right)$, is probably due to synchrotron emission, while the harder part of the spectrum, which has $\alpha \simeq 1$, is due to inverse Compton emission. The blazar shows the long and short-term variability typical of low-frequency peaked BL Lac (LBL): the former is manifested by a gradual decrease in the optical flux from the peak as observed by ground telescopes at the end of March 2004, while the latter is characterized by soft X-ray and optical flares on time scales from a few thousand seconds to few hours. We can follow spectral variations on sub-hour time scales and study their correlation with the flux variability. We find evidence that the peak energy of the time-resolved spectra is increasing with flux. The modeling of the spectral energy distribution compared with archival observations suggests that the long-term variability (from outburst to quiescence or viceversa) could be due to a change in the injected power, while the short-term variability (flares) could be explained with changes in the slope of the distribution of the electrons.
\end{abstract}

Key words. galaxies: BL Lacertae objects: general - galaxies: BL Lacertae objects: individual: S5 0716 + 714 - X-rays: galaxies

\section{Introduction}

The BL Lac object S5 $0716+714$ is one of the most variable blazars with intraday variability at radio, optical, and X-ray wavelengths suggesting a physical origin intrinsic to the source (e.g. Wagner \& Witzel 1995; Wagner et al. 1996; Montagni et al. 2006; Stalin et al. 2006). The optical continuum is so featureless that every attempt to determine the redshift has failed to date. The non-detection of the host galaxy first set a lower limit of $z>0.3$ (Wagner et al. 1996) and more recently one of $z>0.52$ (Sbarufatti et al. 2005).

The variability of this blazar is strong in every energy band: the optical and radio historical behaviour has been recently summarised by Raiteri et al. (2003), while at the other end of the electromagnetic spectrum, the EGRET telescope onboard the Compton Gamma-Ray Observatory (Hartman et al. 1999) detected several times S5 $0716+714$. Interestingly the strongest $\gamma$-ray detection, with a peak flux of $3.2 \pm 0.7 \times 10^{-7} \mathrm{ph} \mathrm{cm}^{-2} \mathrm{~s}^{-1}$ (to be compared with the average EGRET value of $1.8 \pm 0.2 \times$ $10^{-7} \mathrm{ph} \mathrm{cm}^{-2} \mathrm{~s}^{-1}$ ), was recorded in February 1995, the same year as a major optical outburst (with a peak around $R=12.8$ ) and

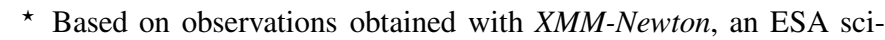
ence mission with instruments and contributions directly funded by ESA Member States and the USA (NASA). small radio variations (cf. Raiteri et al. 2003; see also Ghisellini et al. 1997).

The X-ray observations have shown strong variations with short flares ( $\approx 1000 \mathrm{~s}$ ) detected with ROSAT (Cappi et al. 1994). Broad-band observations with BeppoSAX were performed in 1996 and 1998 during low optical activity (Giommi et al. 1999) and in 2000 (Tagliaferri et al. 2003), triggered by a strong optical outburst, when the blazar displayed an increase of $2.3 R$-band magnitudes in 9 days (cf. Raiteri et al. 2003). In the first two observations, S5 $0716+714$ was at a low flux state in the $0.1-10 \mathrm{keV}$ energy band $\left(3.4 \times 10^{-12} \mathrm{erg} \mathrm{cm}^{-2} \mathrm{~s}^{-1}\right.$ in 1996 and $4.4 \times 10^{-12} \mathrm{erg} \mathrm{cm}^{-2} \mathrm{~s}^{-1}$ in 1998) and was not detected at energies above $10 \mathrm{keV}$. The soft $\mathrm{X}$-rays (below $\approx 2.3 \mathrm{keV}$ ) displayed significant variability in agreement with the optical lightcurves, with the possibility that the optical and X-ray variations were not correlated, but the poor statistics prevented the authors from making firm conclusions (Giommi et al. 1999).

The BeppoSAX observation of 2000 confirmed the findings by Giommi et al. (1999), according to which the soft X-ray spectrum becomes steeper when the source flux increases. The source was also detected in hard X-rays up to $60 \mathrm{keV}$. Again, variability appears to be present only in the soft X-rays (Tagliaferri et al. 2003). 
In April 2004, XMM-Newton and INTEGRAL observed S5 $0716+714$ nearly simultaneously, triggered by an optical outburst that occurred at the end of March. The source was detected in the hard X-ray energy band (Pian et al. 2005). In Sect. 2 we report analysis of the XMM-Newton EPIC camera data and describe the temporal and spectral results; in Sect. 3 we describe the results of the optical observations performed with the XMM-Newton Optical Monitor and with the INTEGRAL Optical Monitor Camera. The latter data were not presented in Pian et al. (2005). In Sect. 4 we describe the multiwavelength energy distribution of the blazar. The results are discussed in Sect. 5.

\section{X-ray data}

The BL Lac object S5 $0716+714$ was observed from 11:07 UT of 4 April 2004 to 02:52 UT of 5 April 2004 (ObsID 0150495601, PI Tagliaferri) as part of a target-ofopportunity observation coordinated with INTEGRAL (Pian et al. 2005). The EPIC MOS1 and MOS2 detectors (Turner et al. 2001) were set in small-window (with thin filter) and full-frame (with thick filter) mode, respectively. The EPIC PN detector (Strüder et al. 2001) was set in timing mode (thin filter), without imaging, but very high time resolution $(0.03 \mathrm{~ms})$ in order to avoid pile-up effects. For the processing, screening, and analysis of the data from the EPIC camera, standard tools were used (XMM SAS v. 6.5, with the latest calibration files, including the patch of August 2005, and HEAsoft Xspec 11.3.21 and Xronos 5.21). To analyse MOS data, we followed the standard procedures described in Snowden et al. (2004), while we adopted the procedure outlined in Brinkmann et al. (2005) for PN (set in timing mode).

The nominal total exposure time was about $60 \mathrm{ks}$; however, the high background reduced the effective exposure of MOS1 and PN to about $30 \mathrm{ks}$. The MOS2 was relatively unaffected by the high background thanks to the use of the thick filter (Fig. 1). To determine the start of high background periods precisely, we integrated the whole detector lightcurve at energies $E>10 \mathrm{keV}$ and excluded the periods with rates $>0.35 \mathrm{c} / \mathrm{s}$ for MOS 1 and $>1 \mathrm{c} / \mathrm{s}$ for PN, as suggested by Kirsh (2005). For MOS2, we adopted a conservative threshold of $0.2 \mathrm{c} / \mathrm{s}$, which allowed us to obtain about $50 \mathrm{ks}$ of good exposure.

\subsection{Time variability}

We extracted the lightcurves from the three detectors of the EPIC camera. The time resolution of MOS1 (small window mode), MOS2 (full frame mode), and PN (timing mode) is $0.3 \mathrm{~s}$, $2.6 \mathrm{~s}$, and $3 \times 10^{-5} \mathrm{~s}$, respectively.

We checked for pile-up effects with the epatplot task of the XMM SAS and found mild pile-up for the MOS2 data (full frame mode), which could be removed by using an annular source region with inner and outer radii of $5^{\prime \prime}$ and $35^{\prime \prime}$, respectively. The MOS1 data (small window mode) are not affected by pile-up, so we adopted a circular region with $35^{\prime \prime}$ radius for the source spectra extraction. The background is extracted from a nearby source-free region of the same size. The source region was centred on the optical position of S5 $0716+714, \alpha=07: 21: 53.4$, $\delta=+71: 20: 36(\mathrm{~J} 2000)$.

For the PN (set in timing mode and therefore without imaging capabilities, cf. Brinkmann et al. 2005, for more details on the analysis of this specific mode), we selected the columns $30 \leq R A W X \leq 47$ for the source and $10 \leq R A W X \leq 21$ for the background. For the latter, we used a smaller size to exclude
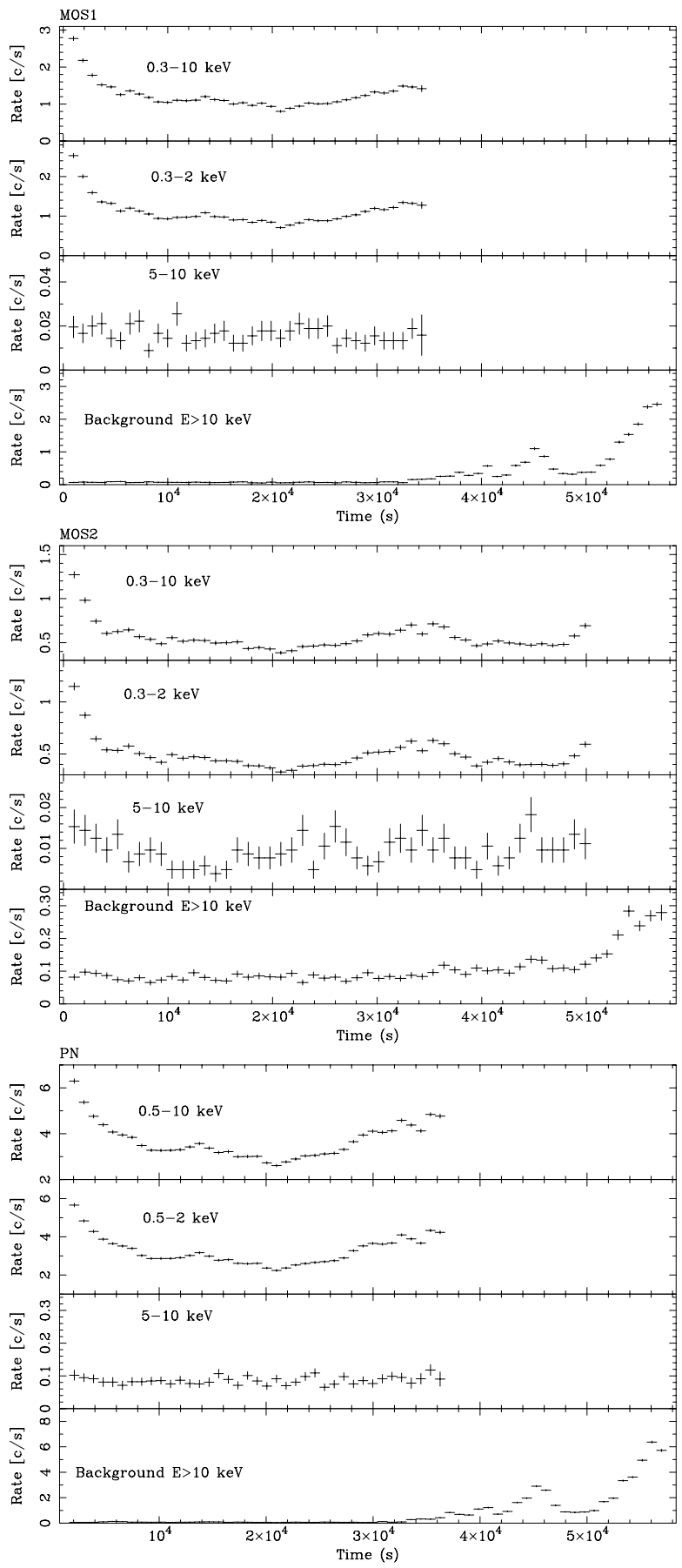

Fig. 1. EPIC MOS1 (top panel), MOS2 (centre panel), and PN (bottom panel) lightcurves. For the two MOS detectors, the four panels indicate: source lightcurve in the energy bands $0.3-10 \mathrm{keV}, 0.3-2 \mathrm{keV}$, and $5-10 \mathrm{keV}$, plus the background lightcurve obtained from the whole detector count rate in the energy band $E>10 \mathrm{keV}$. For the PN detector, the four panels are: source lightcurve in the energy bands $0.5-10 \mathrm{keV}$, $0.5-2 \mathrm{keV}$, and 5-10 keV, plus the background lightcurve obtained from the whole detector count rate in the energy band $E>10 \mathrm{keV}$. The time start is 4 April 2004 at $11^{\mathrm{h}}: 10^{\mathrm{m}}: 00^{\mathrm{s}}$ UTC. Time bins are $1040 \mathrm{~s}$ wide for the MOS2 and $900 \mathrm{~s}$ for MOS1 and PN.

a column containing a hot pixel. The background rates were then properly rescaled before subtracting them from the source-plusbackground rates.

In order to minimize the impact of the background and other spurious effects, we selected only single pixel events 
Table 1. Spectral fits to the EPIC MOS1, MOS2, and PN data $(0.65-10 \mathrm{keV})$ with a fixed absorption column $\left(N_{\mathrm{H}}=3.81 \times 10^{20} \mathrm{~cm}^{-2}\right.$, Dickey \& Lockman 1990). The three time regions selected for time resolved spectral analysis are shown in Fig. 3. The uncertainties in the parameters correspond to a $90 \%$ confidence level for one parameter.

\begin{tabular}{|c|c|c|c|c|c|c|}
\hline Model $^{a}$ & $\Gamma_{1}^{b}$ & $\Gamma_{2}^{c}$ & $E_{\text {break }}{ }^{d}$ & $\tilde{\chi}^{2} /$ d.o.f. ${ }^{e}$ & $F_{\text {soft }}^{f}$ & $F_{\text {hard }}{ }^{g}$ \\
\hline \multicolumn{4}{|c|}{ Whole observation (average) } & \multicolumn{3}{|c|}{$(\mathrm{MOS} 1+\mathrm{MOS} 2+\mathrm{PN})$} \\
\hline PL & $2.61 \pm 0.01$ & & & $1.91 / 1030$ & 1.1 & 0.31 \\
\hline BKPL & $2.78 \pm 0.02$ & $2.09_{-0.05}^{+0.04}$ & $2.0 \pm 0.1$ & $1.08 / 1028$ & 1.2 & 0.41 \\
\hline \multicolumn{4}{|c|}{ Period 1 (0-2.5 ks; high flux $)$} & \multicolumn{3}{|c|}{$(\mathrm{MOS} 1+\mathrm{MOS} 2+\mathrm{PN})$} \\
\hline BKPL & $2.86_{-0.04}^{+0.05}$ & $1.9_{-0.3}^{+0.4}$ & $2.9_{-0.8}^{+0.4}$ & $0.97 / 351$ & 2.0 & 0.41 \\
\hline \multicolumn{4}{|c|}{ Period $2(20-25 \mathrm{ks} ;$ low flux $)$} & \multicolumn{3}{|c|}{$(\mathrm{MOS} 1+\mathrm{MOS} 2+\mathrm{PN})$} \\
\hline BKPL & $2.75 \pm 0.05$ & $1.93_{-0.08}^{+0.07}$ & $1.8_{-0.1}^{+0.2}$ & $1.14 / 545$ & 0.88 & 0.38 \\
\hline \multicolumn{4}{|c|}{ Period 3 (30-36 ks; high flux) } & \multicolumn{3}{|c|}{$(\mathrm{MOS} 2)$} \\
\hline BKPL & $2.83 \pm 0.09$ & $1.9_{-0.7}^{+0.3}$ & $2.6_{-0.4}^{+0.9}$ & $1.07 / 85$ & 1.4 & 0.42 \\
\hline
\end{tabular}

${ }^{a}$ PL, simple power-law model; BKPL, broken power-law model. ${ }^{b}$ Photon index or soft photon index for PL and BKPL models, respectively $\left(f_{E} \propto E^{-\Gamma}\right) .{ }^{c}$ Hard photon index for BKPL. ${ }^{d}$ Break energy for BKPL [keV]. ${ }^{e}$ Reduced $\chi^{2}$ and degrees of freedom. ${ }^{f}$ Unabsorbed flux in the $0.3-2 \mathrm{keV}$ energy band $\left[10^{-11} \mathrm{erg} \mathrm{cm} \mathrm{cm}^{-2} \mathrm{~s}^{-1}\right] .{ }^{g}$ Unabsorbed flux in the $2-10 \mathrm{keV}$ energy band $\left[10^{-11} \mathrm{erg} \mathrm{cm}^{-2} \mathrm{~s}^{-1}\right]$.

$(P A T T E R N==0)$ tagged with $F L A G==0$, except for PN, since in timing mode it accepts only $P A T T E R N \leq 4$ (single and double pixel events).

To investigate the $\mathrm{X}$-ray variability, we built lightcurves in the energy bands $0.3-10 \mathrm{keV}, 0.3-2 \mathrm{keV}$, and 5-10 keV for MOS and $0.5-10 \mathrm{keV}, 0.5-2 \mathrm{keV}$, and 5-10 keV for PN. These values were selected according to the suggestion of the latest EPIC calibration report (Kirsch 2005) and to the source spectral characteristics (see next subsection). With a spectral break around $2-3 \mathrm{keV}$, the selected energy bands are representative of the soft (synchrotron) and hard (inverse Compton) components of the source radiation. The soft X-ray flux shows strong variability (see Fig. 1): it initially decreases by a factor 2.5 in a time scale of few hours, it undergoes a $\approx 10^{4} \mathrm{~s}$ duration flare of about $50 \%$ amplitude, which is fully resolved only by the MOS2. At the end of the MOS2 light curve we note that the flux rises again. Some smaller amplitude and shorter timescale variations are also observed. A formal statistical analysis yields a Kolmogorov-Smirnov and $\chi^{2}$ probability of constancy approaching 0 and a rms $=27 \pm 2 \%$ (MOS2 data, soft band lightcurve, with $2.6 \mathrm{~s}$ bin, with negligible changes depending on the time bin). The hard X-ray emission (5-10 keV) does not show strong variability with $\mathrm{rms} \leq 20 \%$ (upper limit $3 \sigma$ ).

\subsection{Spectral characteristics}

The source counts for the spectral analysis were extracted from the same regions as were used for the lightcurves, while the background counts were collected from a larger $\left(220^{\prime \prime}\right)$ sourcefree region. The spectra were rebinned so that each energy bin contained a minimum of 20 counts and fitted in the $0.65-10 \mathrm{keV}$ energy range (cf. Brinkmann et al. 2005, for PN in timing mode and Kirsch 2005, for MOS and intercalibration problems between MOS and PN). The photon redistribution matrix and the related ancillary file were created appropriately with the rmfgen and arfgen tasks of XMM SAS.

The data from the three detectors were fitted together and the fit parameters are reported in Table 1. The single power-law model does not provide a good fit with evident residuals at high energy. The fit improves a lot with the broken power-law model, which we consider the best-fit model. Figure 2 shows the count spectra obtained with the data of the EPIC camera.

Since the lightcurves show significant variability on different time scales, we selected three time regions corresponding to high

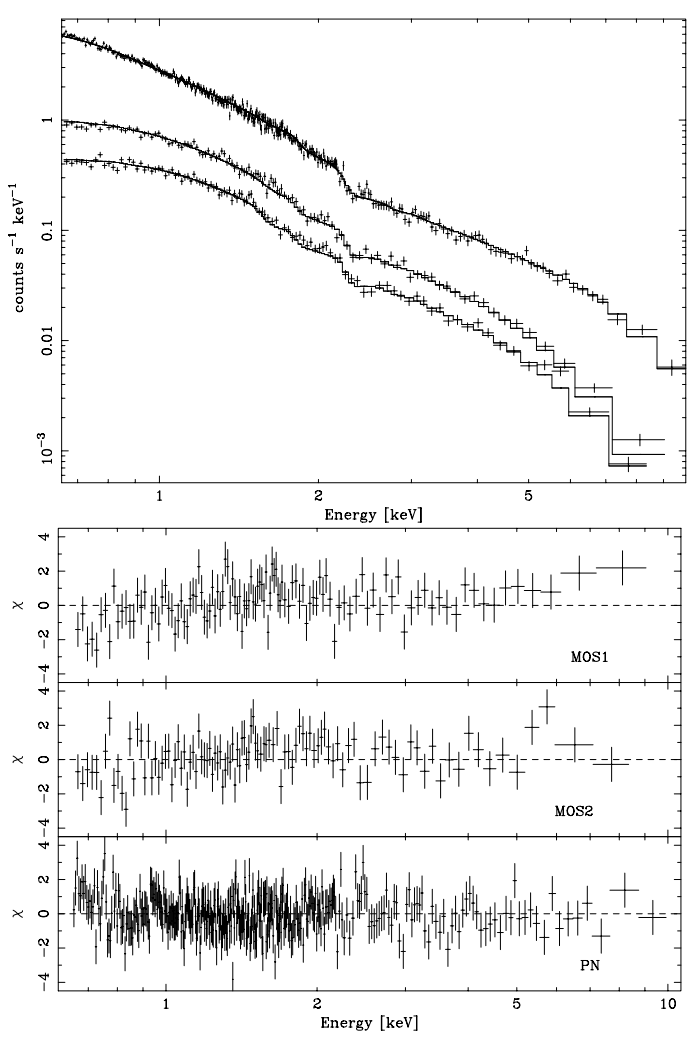

Fig. 2. (Upper panel) EPIC MOS1, MOS2, and PN count spectra for the whole observation with residuals in terms of sigma (lower panel). The solid curves represent the best-fit model (broken power law).

and low flux periods (see Fig. 3) and fitted the extracted spectra with the broken power-law model (the fit with a single powerlaw is not acceptable for any of the three spectra). The parameters of the three fits indicate the typical behaviour of LBL: as the flux rises, the spectrum at low energies becomes softer and the break energy increases, while the hard-band spectral slope remains constant.

\section{Optical data}

Several exposures at different wavelengths were collected by the Optical Monitor (OM, Mason et al. 2001) onboard $X M M-N e w t o n$ during the observation. We reprocessed the 


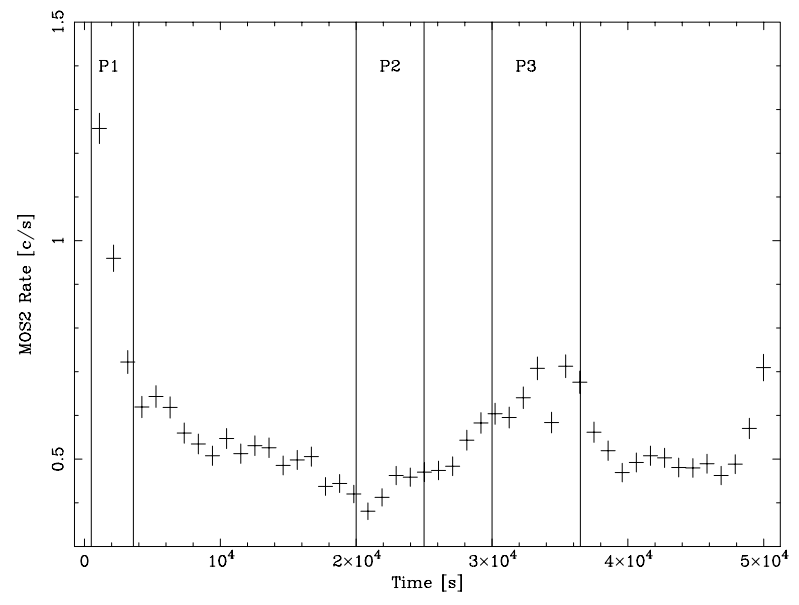

Fig. 3. EPIC MOS2 lightcurve $(0.3-10 \mathrm{keV})$ with the time regions selected for the spectral analysis. The corresponding spectral fits are shown in Table 1.

Table 2. Optical data of S5 $0716+714$ from the Optical Monitor (imaging mode) onboard XMM-Newton. All the exposures were about $1800 \mathrm{~s}$ long. The uncertainties in the parameters are at $1 \sigma$ level.

\begin{tabular}{lcc}
\hline \hline Date/time $^{a}$ & Band $^{b}$ & Magnitude $^{c}$ \\
\hline $04 / 04 / 2004,13: 22$ & $U$ & $12.9 \pm 0.1$ \\
$04 / 04 / 2004,15: 29$ & $U V W 1$ & $12.8 \pm 0.1$ \\
04/04/2004, 18:06 & $U V M 2$ & $13.0 \pm 0.1$ \\
04/04/2004, 20:13 & $V$ & $13.4 \pm 0.1$ \\
04/04/2004, 22:19 & $U$ & $13.0 \pm 0.1$ \\
05/04/2004, 00:26 & UVW1 & $13.0 \pm 0.1$ \\
$05 / 04 / 2004,02: 00$ & UVM2 & $12.6 \pm 0.1$ \\
\hline
\end{tabular}

${ }^{a}$ Observation start date (dd/mm/yyyy) and time (UT). ${ }^{b}$ Band, with effective wavelength of $543 \mathrm{~nm}$ for $V, 344 \mathrm{~nm}$ for $U, 291 \mathrm{~nm}$ for $U V W 1$, and $231 \mathrm{~nm}$ for $U V M 2 .{ }^{c}$ Observed magnitude (i.e. not dereddened).

OM data with the SAS version also used for the EPIC analysis, which gives the observed magnitudes already converted from $\mathrm{OM}$ count rates (the region used for the photometry has a radius equal to 3 times the $F W H M$ of the PSF, which in turn is $\approx 2^{\prime \prime}$ for $\mathrm{UV}$ and $\approx 1^{\prime \prime}$. 4 for optical filters). The results are summarised in Table 2. These measurements were used in the spectral energy distribution (see the next section) and the magnitudes were dereddened by using the extinction curve by Cardelli et al. (1989) with $A_{\mathrm{V}}=0.102$ (Dickey \& Lockman 1990) and converted into flux by using the standard formulae (e.g. Zombeck 1990).

The Optical Monitor Camera (OMC, Mas-Hesse et al. 2003) onboard the INTEGRAL satellite provides optical data with a standard Johnson $V$ filter simultaneously with the high-energy data of the on-axis source. The OMC PSF is $25^{\prime \prime}$-sized (FWHM), and we checked the USNO B1 Catalog (Monet et al. 2003) for contaminating sources inside a region with twice this radius. We found 4 sources, in addition to the blazar, with $B$ magnitudes around 20 exhibiting no significant variability between the 2 epochs at which the USNO survey was performed, so that their contribution can be neglected.

The $V$ filter lightcurve of S5 $0716+714$ (Fig. 4), obtained from the preprocessed data of the OMC public archive ${ }^{1}$,

\footnotetext{
1 http://sdc.laeff.esa.es/omc/. Based on data from the INTEGRAL/OMC Archive at LAEFF, processed by ISDC. INTEGRAL is an ESA mission with instruments and a science data centre funded by ESA member states (especially the PI countries: Denmark, France,
}

was dereddened with $A_{\mathrm{V}}=0.102$ (Dickey \& Lockman 1990) and converted into flux by using the standard formulae (e.g. Zombeck 1990).

INTEGRAL observed the blazar twice between 10-17 November 2003 (ObsID 0120177, PI Wagner) and between 2-7 April 2004 (ObsID 0220049, PI Pian). During the November 2003 observation, S5 $0716+714$ was in an optically faint state $(R=14.17-13.64)$ and there was no detection at hard X-rays (Ostorero et al. 2006; see also Beckmann et al. 2006).

In the second INTEGRAL observation (April 2004), the optical flux was higher, consistent with the one measured from the XMM-Newton OM (see Fig. 4) and with hard X-rays detection (Pian et al. 2005). A general decrease over the observation duration is modulated by shorter time scale flares, with flux variations of the order of $30 \%$ (Fig. 4).

\section{Spectral energy distribution}

In Fig. 5 we report the spectral energy distributions of S5 $0716+714$ constructed with historical data (see Tagliaferri et al. 2003) and with the INTEGRAL and XMM-Newton data acquired in April 2004. We adopted the model described in Ghisellini et al. (2002), to which we refer the reader for a detailed explanation of the parameters, and assumed a redshift $z=0.3$ for $\mathrm{S} 50716+714$, in order to compare the present results with similar modeling performed on the past observations (cf. Table 4 in Tagliaferri et al. 2003).

The model envisages synchrotron radiation and inverse Compton scattering as responsible for the spectral emission at low (radio to UV) and high (X- and gamma-rays) frequencies. The geometry of the emitting region is a cylinder with radius $R=2 \times 10^{16} \mathrm{~cm}$ and height $\Delta R^{\prime} \approx R / \Gamma=1.3 \times 10^{15} \mathrm{~cm}$. The bulk Lorentz factor is $\Gamma=15$ and the viewing angle $\theta=3.4^{\circ}$, implying a Doppler factor $\delta=16.7$. The magnetic field is $3 \mathrm{G}$ and the electron distribution is assumed to be $N(\gamma) \propto \gamma^{-n}$. For the present case there are three different distribution regions:

$-N(\gamma) \propto \gamma^{-2}$, for $\gamma<\gamma_{\text {break }}$

$-N(\gamma) \propto \gamma^{-2.9}$, for $500=\gamma_{\text {break }}<\gamma<\gamma_{\text {cool }}$;

- $N(\gamma) \propto \gamma^{-3.9}$, for $1424=\gamma_{\text {cool }}<\gamma<\gamma_{\max }=3.7 \times 10^{4}$;

where $\gamma_{\text {cool }}$ is the Lorentz factor for which the radiative cooling time is equal to $\Delta R^{\prime} / c$. The injected power in the comoving frame is $L=4 \times 10^{42} \mathrm{erg} / \mathrm{s}$.

The present parameters imply minimal changes with respect to the model parameters of the 1996 observation when the source was in low activity, except for the injected power, which in 1996 was about half the present value $\left(2.2 \times 10^{42} \mathrm{erg} / \mathrm{s}\right)$, and for some changes in $\gamma_{\max }$. For the observation in 2000, when the source was active, the model requires similar parameters and an injected power of $5 \times 10^{42} \mathrm{erg} / \mathrm{s}$, very similar to the present case. For more details on the model parameters of the previous BeppoSAX observations see Tagliaferri et al. (2003).

\section{Discussion}

XMM-Newton observed S5 0716+714 in April 2004 after an optical outburst and detected it in a high soft X-ray state. The X-ray flux in the $0.1-2 \mathrm{keV}$ band is about a factor of 5 and a factor of 2 higher than detected by BeppoSAX in 1996 and 1998, and

Germany, Italy, Switzerland, Spain), the Czech Republic, and Poland, and with the participation of Russia and the USA. 


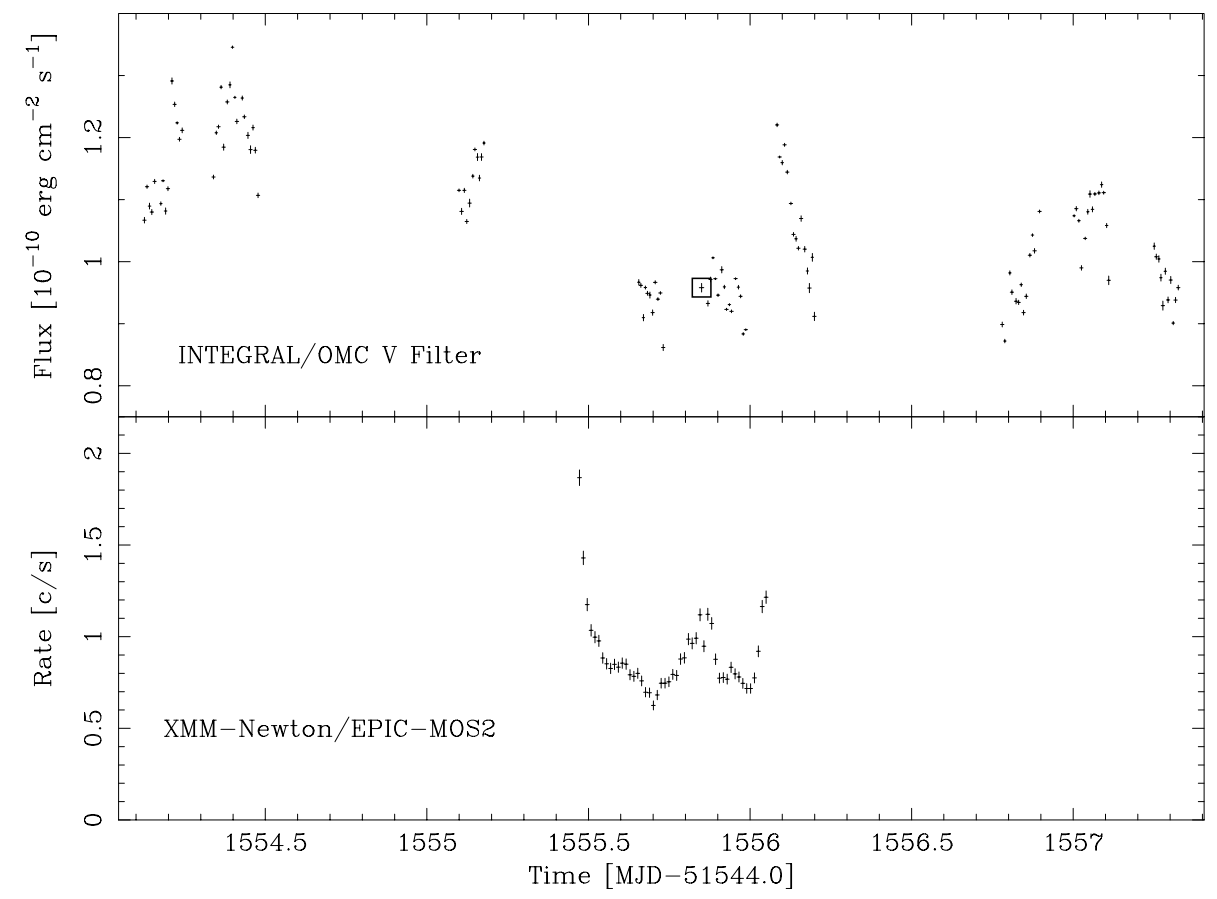

Fig. 4. (Upper panel) INTEGRAL/OMC $V$ filter lightcurve in the period 3-6 April 2004. The square encloses the point from the OM onboard $X M M-N e w t o n$ (see Table 2). (Lower panel) Simultaneous XMM-Newton data from EPIC-MOS2 in the 0.3-10 keV energy band.

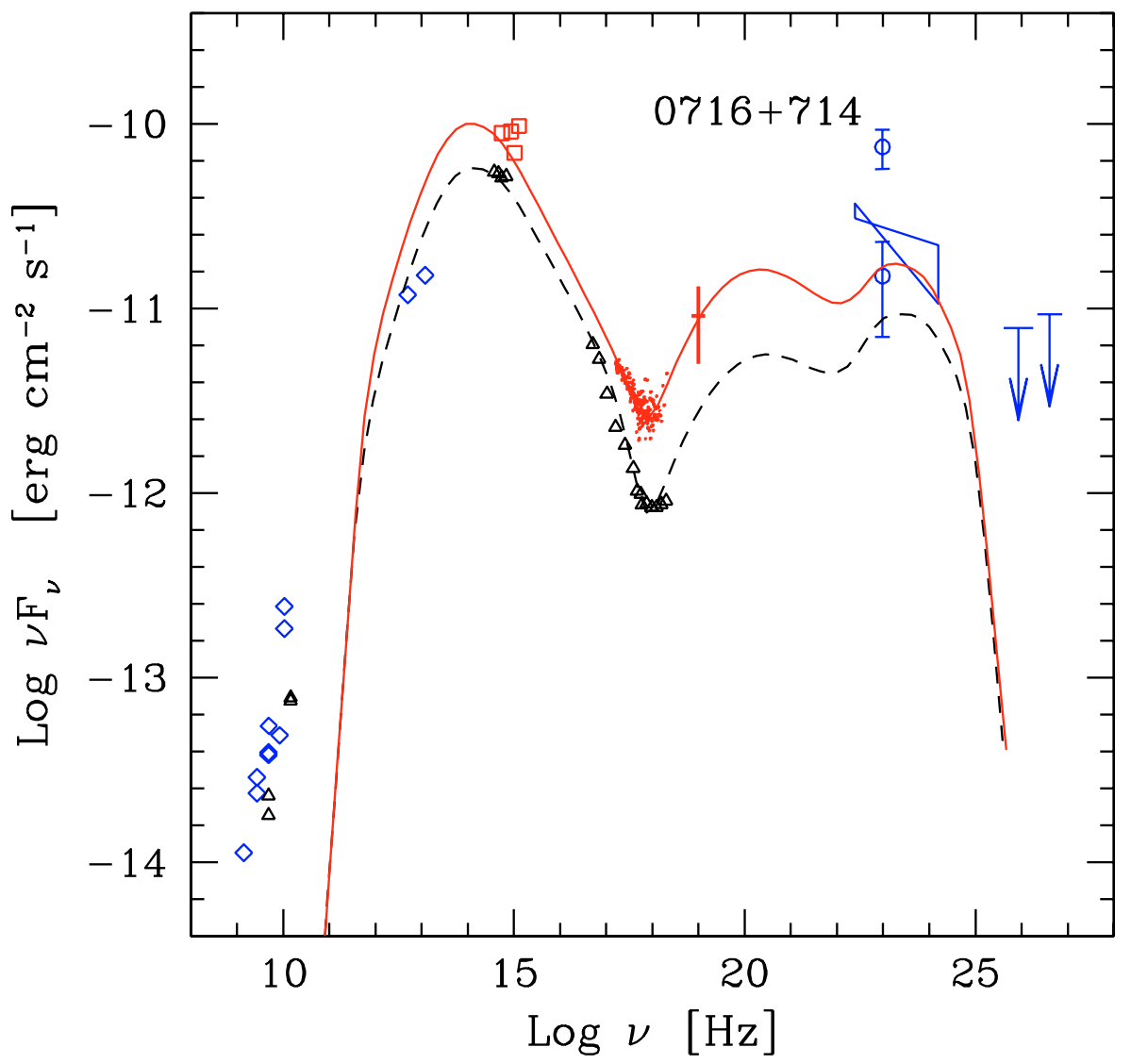

Fig. 5. Spectral energy distributions of S5 $0716+714$ at various epochs. The cross, points, and open squares represent simultaneous INTEGRAL/ISGRI (Pian et al. 2005) and XMM-Newton EPIC and OM (present work) observations in April 2004, respectively. The triangles represent BeppoSAX, optical, and radio nearly simultaneous observations in 1996 (Giommi et al. 1999). Non simultaneous EGRET data are also reported (Hartman et al. 1999), referring to the minimum, maximum, and average flux (open circles). The latter also includes the photon index interval. Radio data (diamonds) and $\mathrm{TeV}$ upper limits are also non simultaneous (see Tagliaferri et al. 2003). The lines represent the model by Ghisellini et al. (2002) adapted to fit the April 2004 (continuous line) and November 1996 (dashed line) energy distributions. 

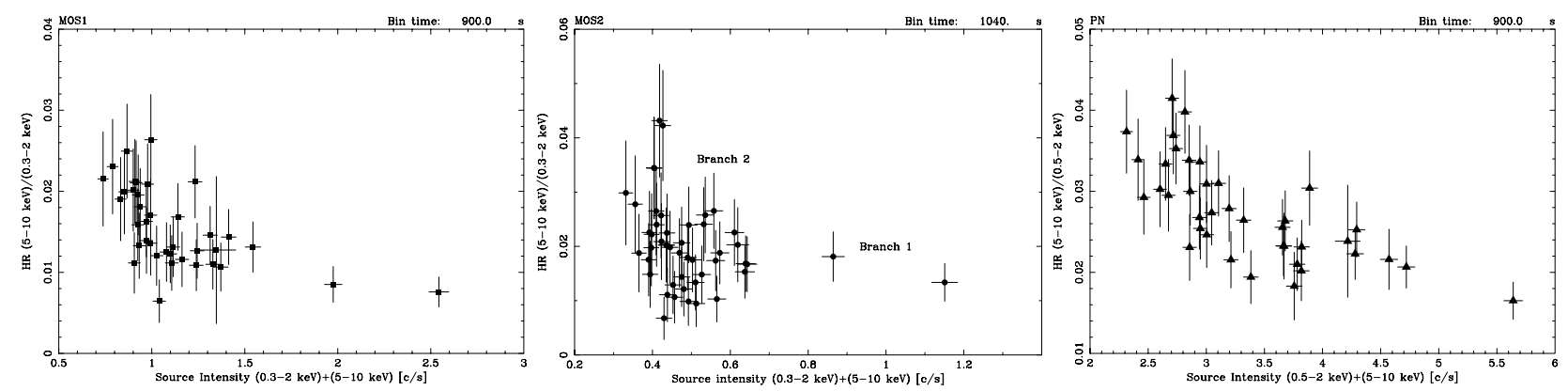

Fig. 6. Source intensity versus hardness ratio - defined as (5-10)/(0.3-2) keV - for MOS1 (left panel), MOS2 (centre panel), and for PN (right panel), but in the energy bands $(0.5-2)$ and $(5-10) \mathrm{keV}$.

in 2000 , respectively. The $2-10 \mathrm{keV}$ flux appears stabler: it is comparable to the 2000 level and a factor 2 higher than in 1996 (Giommi et al. 1999; Tagliaferri et al. 2003). The hour time-scale variability of the X-ray flux observed during the present observation confirms that the soft X-ray variability has larger amplitude than the hard X-ray variability (Fig. 1). This suggests that different emission components are responsible for the soft and hard $\mathrm{X}$-rays, as already proposed by the previous authors.

Accordingly, the X-ray spectrum is not consistent with a single power-law, and is rather described by a broken power-law. The soft X-ray emission represents the higher energy tail of a synchrotron spectrum, and is therefore highly variable, while the hard X-ray emission, which has a flat spectrum, detected up to $60 \mathrm{keV}$ (Tagliaferri et al. 2003; Pian et al. 2005), is less variable, as it originates from inverse Compton scattering of relativistic electrons off modestly variable infrared and radio synchrotron radiation. The X-ray hour time scale spectral variations support this interpretation by providing evidence for the shift of the break energy toward higher energies during flares. In order to corroborate our short time scale spectral findings, we have also constructed intensity-hardness ratio plots (Fig. 6). These display the typical characteristics of other well known Low-Energy Peaked BL Lacs (LBL), like BL Lac itself (Ravasio et al. 2002, 2003) and ON 231 (Tagliaferri et al. 2000). The trend is very close to the BeppoSAX behaviour (cf. Fig. 4 in Giommi et al. 1999), with high hardness at low fluxes.

The samplings of the XMM-Newton EPIC and INTEGRAL OMC lightcurves do not coincide exactly and, particularly for the optical data, several time gaps are present, therefore it is difficult to establish a correlation between the two. The decreasing trend of the OMC lightcurve suggests that S5 $0716+714$ is returning to its quiescence state after the outburst at the end of March 2004. The short flare at the end of the XMM-Newton EPIC lightcurve seems to have a counterpart in the optical (Fig. 4), but the gap at the beginning of the optical flare does not allow us to evaluate the real start of the event.

We note two types of activity of S5 $0716+714$, labelled in Fig. 6 (centre panel) with "Branch 1" and "Branch 2", respectively: the first type (Branch 1) is likely to be related to the activity following the major optical outburst that occurred at the end of March 2004 (these points indeed refer to the very beginning of the X-ray lightcurves shown in Fig. 1). We already noted that, as shown in Fig. 4, the optical flux measured by OMC is gradually declining. Moreover, Pian et al. (2005) report that the $30-60 \mathrm{keV}$ flux during the first $\approx 80 \mathrm{ks}$ of the INTEGRAL observation is greater than the average over the whole obsevation. Therefore, although the position of the branch 1 in the intensityhardness ratio plots favours the hypothesis that can be the final tail of the outburst, we cannot exclude that we are seeing the decaying part of a major flare occurred during the gradual declining of the source after the outburst.

The second type of activity (Branch 2) is linked to the short flares randomly occurring during the long term activity. Similar behaviour has been already observed in other BL Lac objects, like the high-energy peaked BL Lac (HBL) Mkn 421 (e.g. Sembay et al. 2002; Brinkmann et al. 2003; Ravasio et al. 2004; Brinkmann et al. 2005) or PKS 2155-304 (Zhang et al. 2006). However, it is worth noting the different origins of this behaviour: in HBL, the X-ray emission is due to the synchrotron radiation, while in LBL there is competition between synchrotron and inverse Compton. The HBL display variability both in the soft and hard X-ray energy bands, with more significant variability at high energy: higher fluxes correspond to harder spectra, reflecting changes in the particle injection. On the other hand, the LBL are generally variable only in the soft X-rays, while the hard X-ray energy band shows little or no variability, so the hardness ratio reflects the dominance of synchtrotron relative to inverse Compton radiation.

As known for other LBL, the soft X-rays' significant variability and the short flares in S5 $0716+714$ are due to the most energetic electrons of the synchrotron spectrum, cooling much faster than the electrons producing the inverse Compton emission, which are likely to be at the low end of the distribution. The gradual decay afterburst is instead likely to be attributed to the escape of electrons from the processing region or to a decrease in the soft seed photons or both.

Instruments with higher senstivity, particularly in the hard $\mathrm{X}$-ray and $\gamma$-ray domains, are necessary to follow the evolution of the source completely. The next generation of satellites operating in the $\gamma$-ray energy band, like GLAST ${ }^{2}$, and in the hard X-ray band, like SIMBOL-X (Ferrando et al. 2005), should provide the optimal temporal resolution for monitoring the shortterm blazar variability, which appears to be the key to understand the mechanisms at work in this class of sources.

Acknowledgements. L.F. wishes to thank R. Gonzalez Riestra of XMM-Newton SOC for discussion of EPIC background and P. Giommi for discussion of previous BeppoSAX observations. Thanks also to the anonymous referee for helpful criticism that improved the manuscript. This work was partially supported by the Italian Space Agency (ASI) under contract I/R/046/04.

\section{References}

Beckmann, V., Gehrels, N., Shrader, C. R., \& Soldi, S. 2006, ApJ, 638, 642 Brinkmann, W., Papadakis, I. E., den Herder, J. W. A., \& Haberl, F. 2003, A\&A, 402, 929

\footnotetext{
2 http://www-glast. stanford.edu
} 
Brinkmann, W., Papadakis, I. E., Raeth, C., Mimica, P., \& Haberl, F. 2005, A\&A, 443,397

Cappi, M., Comastri, A., Molendi, S., et al. 1994, MNRAS, 271, 438

Cardelli, J. A., Clayton, G. C., \& Mathis, J. S. 1989, ApJ, 345, 245

Dickey, J. M., \& Lockman, F. J. 1990, ARA\&A, 28, 215

Ferrando, P., Goldwurm, A., Laurent, P., et al. 2005, Proc. SPIE, 5900, 195

Ghisellini, G., Villata, M., Raiteri, C. M., et al. 1997, A\&A, 327, 61

Ghisellini, G., Celotti, A., \& Costamante, L. 2002, A\&A, 386, 833

Giommi, P., Massaro, E., Chiappetti, L., et al. 1999, A\&A, 351, 59

Hartman, R. C., Bertsch, D. L., Bloom, S. D., et al. 1999, ApJS, 123, 79

Kirsch, M. 2005, EPIC status of calibration and data analysis, XMM-SOC-CALTN-0018, v. 2.4, 11 Feb. 2005

Mas-Hesse, J. M., Gimènez, A., Culhane, J. L., et al. 2003, A\&A, 411, L261

Mason, K. O., Breeveld, A., Much, R., et al. 2001, A\&A, 365, L36

Monet, D. G., Levine, S. E., Casian, B., et al. 2003, AJ, 125, 984

Montagni, F., Maselli, A., Massaro, E., et al. 2006, A\&A, 451, 435

Ostorero, L., Wagner, S. J., Gracia, J., et al. 2006, A\&A, 451, 797

Pian, E., Foschini, L., Beckmann, V., et al. 2005, A\&A, 429, 427

Raiteri, C. M., Villata, M., Tosti, G., et al. 2003, A\&A, 402, 151
Ravasio, M., Tagliaferri, G., Ghisellini, G., et al. 2002, A\&A, 383, 763 Ravasio, M., Tagliaferri, G., Ghisellini, G., et al. 2003, A\&A, 408, 479 Ravasio, M., Tagliaferri, G., Ghisellini, G., \& Tavecchio, F. 2004, A\&A, 424, 841

Sbarufatti, B., Treves, A., \& Falomo, R. 2005, ApJ, 635, 173

Sembay, S., Edelson, R., Markowitz, A., et al. 2002, ApJ, 574, 634

Snowden, S., Still, M., Harrus, I., et al. 2004, an introduction to XMM-Newton data analysis, Version 2.01, 23 July 2004

Stalin, C. S., Gopal-Krishna, Sagar, R., et al. 2006, MNRAS, 366, 1337

Strüder, L., Briel, U., Dennerl, K., et al. 2001, A\&A, 365, L18

Tagliaferri, G., Ghisellini, G., Giommi, P., et al. 2000, A\&A, 354, 431

Tagliaferri, G., Ravasio, M., Ghisellini, G., et al. 2003, A\&A, 400, 477

Turner, M. J., Abbey, A., Arnaud, M., et al. 2001, A\&A, 365, L27

Wagner, S. J., \& Witzel, A. 1995, ARA\&A, 33, 163

Wagner, S. J., Witzel, A., Heidt, J., et al. 1996, AJ, 111, 2187

Zhang, Y. H., Treves, A., Maraschi, L., Bai, J. M., \& Liu, F. K. 2006, ApJ, 637, 699

Zombeck, M. V. 1990, Handbook of space astronomy and astrophysics (Cambridge: Cambridge University Press) 